\title{
Second-year Outcomes of Implementing Antimicrobial Stewardship Program in a Tertiary Pediatric Hospital
}

\author{
Iman Khorshidi-Malahmadi ${ }^{1}$, Setareh Sima ${ }^{1}$, Najmeh Ansari ${ }^{2}$ and Azadeh Moghaddas (id ${ }^{1,{ }^{*}}$ \\ ${ }^{1}$ Department of Clinical Pharmacy, Faculty of Pharmacy, Isfahan University of Medical Sciences, Isfahan, Iran \\ ${ }^{2}$ Clinical Microbiology Laboratory, Imam Hussein Hospital,Isfahan, Iran \\ "Corresponding author: Department of Clinical Pharmacy, Faculty of Pharmacy, Isfahan University of Medical Sciences, Isfahan, Iran. Email: moghaddas@pharm.mui.ac.ir
}

Received 2021 August 04; Revised 2021 October 02; Accepted 2021 October 03.

\begin{abstract}
Background: The increasing use of antimicrobial agents and resistance is becoming a serious problem in pediatric patients. Hence, antimicrobial stewardship program (ASP) is implemented to lessen the consequences.

Objectives: This report aimed to demonstrate the impact of ASP on antimicrobials utilization in a referral and tertiary pediatric hospital in Isfahan, Iran.

Methods: We launched an ASP for inpatients in March 2018 at the Imam Hussain Hospital, which is a 186-bed pediatrics hospital. Data was collected by ASP multidisciplinary team and using hospital records during February 2017-January 2018 (pre-intervention) and February 2019-January 2020 (post-intervention) periods. Consumption data were expressed as defined daily doses (DDDs) per 100 patient-days (PD). Independent sample t-test and paired t-test were used to assess the significance of differences. The rates of antimicrobials resistance for the most common hospital pathogens were also tracked.

Results: Utilization of total antimicrobials decreased meaningfully by 12.41\% (from 62.11 DDDs/100 PD in February 2017-January 2018 to 54.40 DDDs/100 PD in February 2019-January 2020; $\mathrm{P}=0.024$ ). The results showed that the reduction in non-restricted antimicrobials was less (5.43\%) than restricted antimicrobials (27.6\%).

Conclusions: According to our results, the use of antimicrobials, especially the broad-spectrum ones, and the health burden costs significantly decreased after implementing ASP. Thus, continuous monitoring and educational programs are recommended to reduce the negative effects of using antimicrobials.
\end{abstract}

Keywords: Antibiotic Stewardship, Inpatients, Pediatrics, Broad-Spectrum Antibiotic

\section{Background}

Antimicrobial stewardship is a program for ensuring cost-effective antimicrobials therapy and reducing antimicrobial resistance (AMR) rate in hospitalized patients (1). Many successful antimicrobial stewardship programs (ASP) have been implemented in adult patients to improve antimicrobials prescription accuracy, lower local resistance rates, and reduce healthcare costs (2-6). In several adult studies, ASP was successful in assisting physicians in selecting the appropriate antimicrobials, as well as the appropriate dose and duration of therapy. However, few pediatric hospitals have adopted the ASP system. The number of studies conducted on the effects of ASP in children is scarce (7-9). Since antimicrobial agents are among the most widely prescribed medications in hospitalized children (10), implementing an effective ASP for pediatric pop- ulations is essential. Misuse and long-term exposure to antimicrobial agents such as vancomycin, meropenem, etc., in pediatric centers could induce multidrug resistance bacterial species (such as vancomycin-resistant Enterococcus spp. and carbapenem-resistant Gram-negative bacteria) and increase healthcare costs.

\section{Objectives}

In March 2018, an ASP was developed in our pediatric center to monitor the utilization and costs of antimicrobial agents and reduce multidrug-resistant bacteria. Accordingly, this study aimed to investigate how an ASP was able to affect antimicrobial agents' utilization, costs, and AMR in the second year of implementation in a pediatric hospital in Isfahan, Iran. 


\section{Methods}

\subsection{Study Design}

The current prospective cross-sectional study was performed by analyzing the results of hospital data before and after ASP implementation at Imam Hussein Hospital in Isfahan, Iran. The hospital is a university-affiliated referral center for pediatric patients. Data was collected from October 2020 to January 2021. The Infection Control Committee of the hospital introduced an ASP for inpatients to encourage guideline-concordant care for antimicrobials utilization to reduce rates of using antimicrobials, as well as antimicrobial resistance $(11,12)$. A multidisciplinary ASP non-dedicated staff team including two infectious diseases physicians, two pharmacists (a hospital pharmacist and a clinical pharmacist), two consultant clinical microbiologists, and two infection control nurses collaborated with some nominated clinicians who worked in clinical departments.

The protocol of the study was approved by the Ethics Committee of the hospital. We extracted baseline information about patient days (PD) and admissions from hospital administration center. Patient days was defined as the total number of patients occupying beds in a long-term care facility for all days in the calendar period for which an assessment was being reported and paid. The data were analyzed based on the anatomic therapeutic chemical (ATC) classification system of the World Health Organization (WHO) (13). We measured the utilization of antimicrobials based on defined daily doses (DDD) per 100 PD. According to the WHO guideline, although DDD for pediatrics is not accurate due to variability of children doses, weights, and difficulty to identify these parameters, general DDD could be a standardized measuring instrument for overall dose comparisons (13).

In addition, the AMR data for the pathogens that were predominate in the hospital (Enterococcus spp., Escherichia coli, Klebsiella spp., Enterobacter spp., Citrobacter spp., Pseudomonas spp., Acinetobacter baumannii, Staphylococcus spp., Streptococcus spp.) was collected from all clinical specimens' records (blood, urine, cerebrospinal fluid, dialysis fluid, abscess wound, and bronchoalveolar lavage), which was extracted from WHONET software 2020 (Boston, MA, USA). WHONET is an effective computerized microbiology laboratory data management and analysis program that can provide guidance for empirical therapy of infections, alert clinicians about antimicrobial resistance, and help to make drug-policy decisions and perform preventive measures.

\subsection{Antimicrobial Stewardship Program}

In March 2018, the Infection Control Committee decided to select several broad-spectrum antimicrobials and identified them as "restricted antimicrobials agents," and implemented an ASP for these antimicrobials to control their consumption. The main technique used in implementing ASP was prospective audit and feedback, which included contact and feedback among an infectious disease physician and the prescribers (14). In accordance with the ASP, all specialists who had prescribed broad-spectrum antimicrobials agents such as carbapenems, vancomycin, teicoplanin, colistimethate sodium, and linezolid were expected to seek advice from an infectious disease specialist within 72 hours of the antimicrobial's administration to ensure that the treatment was optimized based on patients' data and results of microbiological tests. Expert assistance (infectious disease specialist and pharmacists) was available on a daily basis, but not 24 hours a day. For emphasis, two nurses were required to sign the order for these antimicrobials selections. Within 72 hours, blood cultures were sent to a clinical microbiologist.

The ASP team used small-group education to emphasize that it is important to notice antimicrobial stewardship and discontinue surgical prophylaxis in a timely manner (15). The team also ensured that ASP was implemented properly by implementing pharmacists' audit in hospital units on a regular basis. The team focused on educating prescribers through disseminating educational materials, holding meetings, and sending verbal and written reminders to improve their assistance to ASP. Finally, data on antimicrobials utilization were checked regularly to recognize strength and weaknesses, and monitor the effectiveness of the treatments and cost reduction programs after implementing ASP.

\subsection{Statistical Analysis}

The paired t-test and independent samples $t$-test were applied to analyze differences in antimicrobials utilization before the intervention (February 2017-January 2018) and after the intervention (February 2019-January 2020). The chi-square test was used to analyze the variations in AMR before and after the intervention. A P-value less than 0.05 was considered as statistically significant. All statistical analysis was carried out by Statistical Package for the Social Sciences (SPSS) software version 23 (SPSS Inc., Chicago, IL, USA). 


\section{Results}

Table 1 shows the antimicrobials utilization in DDDs per 100 PD before and after the intervention. Total antimicrobials utilization decreased by $12.41 \%$ (from 62.11 DDDs/100 PD to 54.40 DDDs per 100 PD; $\mathrm{P}=0.024)$. The reduction was lower in non-restricted antimicrobial agents (5.43\%) than restricted ones (27.6\%) (Table 2). According to our findings, the use of vancomycin and carbapenems decreased substantially (Table 1 ). Vancomycin use, in particular, decreased from 11.84 DDDs/100 PD before the intervention to 8.29 DDDs/100 PD after the intervention (29.98\%, $\mathrm{P}<0.001)$, while prescription of carbapenems decreased from 6.78 to 4.55 (32.89\%, P $<0.001)$. On the other hand, the use of antibiotics such as teicoplanin and colistin did not show any significant reduction (teicoplanin 0.4 and colistin 0.17 DDDs/100PD before and after the intervention, respectively).

Linezolid was the only restricted antimicrobial agent utilization of which increased after the intervention; however, overall linezolid prescription in our hospital was low and negligible. Table 2 shows the approximate average cost reductions of antimicrobial agents per 100 PD before and after the intervention. The changes in antimicrobial prices were scant during the time of ASP implementation. Despite a decline in DDD/100 PD for total administrative antimicrobial agents, the overall antimicrobial agents' cost increased from 14873833 to 15482933 rials/100 PD (+4.09\%). In terms of pre-and post-intervention resistance rates, our data for the second-year intervention revealed a reduction in vancomycin-resistant enterococci (VRE) from $4.80 \%$ to $2.60 \%(\mathrm{P}=0.024)$ and linezolid-resistant Staphylococcus spp. and Streptococcus spp. from $2.20 \%$ to $1.80 \%(\mathrm{P}=0.225)$. Importantly, meropenem and imipenem resistance rates declined significantly for Gram negative microorganisms from $8.90 \%$ to $7.60 \%(\mathrm{P}=0.017$ ) and $25 \%$ to $14.40 \%$ ( $\mathrm{P}=$ 0.006), respectively. Linezolid-resistant Staphylococcus sp. and Streptococcus sp. decreased from $2.20 \%$ to $1.80 \%$ ( $\mathrm{P}=$ 0.225). Other AMR did not differ significantly from the baseline after the implementation of ASP (Table 1).

\section{Discussion}

Our study aimed to assess the impact of ASP implementation on broad-spectrum antimicrobials administration in one of the largest and referral pediatric hospitals in Isfahan, Iran. According to previous studies, 3.4 - 3.5\% of antimicrobial prescriptions in pediatric patients were noted to be unnecessary and over-prescribed $(16,17)$. Due to inappropriate prescription and consumption of antibiotics, the number of multidrug resistant pathogens like pseudomonas aeroginosa is increasing, and critical restrictions like implementing practical guide antibiotic stewardship is essential for managing this problem $(18,19)$. In a primary overview of our hospital documents, we figured out that expensive and broad-spectrum antimicrobial agents had been overused, which could lead to antibiotic-resistant infections and increase the economic burden of healthcare system. Furthermore, for the first time in our center, we decided to introduce the ASP for managing the utilization of several broad-spectrum antibiotics.

Anvarinejad et al. reported a decline in antibioticresistant microorganisms (20). They found that for controlling the rate of resistant microorganisms, clinicians should consider culture specimens before initiation of antibiotic therapy and supervise the prescribed antibiotics periodically. The results of our study demonstrated that there was a substantial decrease in overall antimicrobial utilization after two years of intervention (excluding linezolid) because of cooperation of majority of prescribers with ASP. Similar to our findings, Pakyz et al. (21) noted that linezolid and macrolide antibiotics utilization increased dramatically while other antimicrobial utilization remained unchanged after ASP implementation.

The threat of AMR is reaching an alarming rate, while the situation is worsening in developing countries due to frequent abuses of antimicrobials (1). It has been demonstrated that even appropriate and justified use of antibiotics can contribute to the development of AMR; thus, widespread and excessive use makes the situation much worse. On the other hand, AMR is a multi-factorial problem, which can be influenced by infection control strategies such as ASP. For instance, a recent publication demonstrated that implementing ASP can lead to rational use of antibiotics, reduce antimicrobial resistance, as well as improve patients' overall outcome (22). Our study also indicated that ASP implementation could reduce broad-spectrum antibiotics resistance in numerous hospital pathogens such as VRE. However, some studies reported that implementing an ASP had no effects on changing antimicrobials resistance rate (23).

It is worth mentioning that, although ASP was effectively performed in our study, due to the sanctionsinduced inflation, the costs of antimicrobial agents increased dramatically; so, we did not witness any cost savings for antimicrobials after the intervention. However, 


\begin{tabular}{|c|c|c|c|c|}
\hline \multirow{2}{*}{ Antibiotics } & \multicolumn{2}{|c|}{ DDDs/10o Patient Days } & \multirow{2}{*}{ Percentage Change } & \multirow{2}{*}{ P-Value } \\
\hline & $2019-2020$ & 2017-2018 & & \\
\hline Vancomycin & 8.29 & 11.84 & -29.98 & 0.033 \\
\hline Carbapenems & 4.55 & 6.78 & -32.89 & 0.001 \\
\hline Teicoplanin & 0.44 & 0.43 & +2.32 & NS \\
\hline Linezolid & 0.64 & 0.26 & +146.15 & 0.001 \\
\hline Colistimethate sodium & 0.17 & 0.17 & 0 & NS \\
\hline \multicolumn{5}{|c|}{$\begin{array}{l}\text { Table 2. Estimated Reductions in Total Antibiotics Utilization and Costs Before the Intervention February 2017-January 2018) and After the Intervention (February 2019-Januar } \\
\text { 2020) }{ }^{\mathrm{a}}\end{array}$} \\
\hline Measure & & & $2019-2020$ & Reduction (\%) \\
\hline Total hospital days & & & 49664 & -0.63 \\
\hline Mean length of hospital stay (days) & & & 3.02 & +20.8 \\
\hline Restricted antibiotics DDDs/100 PD & & & 14.09 & -27.6 \\
\hline Other antibiotics DDDs/10o PD & & & 40.31 & -5.43 \\
\hline Total antibiotics DDDs/100 PD & & & 54.40 & -12.41 \\
\hline $\begin{array}{l}\text { Restricted antibiotics' cost, } \mathbf{R} / 100 \\
\text { PD }\end{array}$ & & & 8216272 & -18.31 \\
\hline $\begin{array}{l}\text { Non-restricted antibiotics' cost, } \\
\text { R/100 PD }\end{array}$ & & & 7266661 & +50.91 \\
\hline Total antibiotics' cost, R/10o PD & & & 15482933 & +4.09 \\
\hline
\end{tabular}

Abbreviations: DDDs, daily defined doses; R, rials.

${ }^{a}$ Prices were computed in rials

the overall cost of antimicrobials could be declined by $5.2 \%$ in a normal situation. In the second year of ASP implementation, the cost of restricted antimicrobials was reduced by $18.31 \%$, which was critical in Iran's current financial situation. The cost saving results demonstrated that hiring full-time infectious disease specialists and clinical microbiologists minimized antimicrobial costs and wellcompensated the burden of hiring $\operatorname{staff}(4,24,25)$. In addition, the leaders of ASP, especially clinical microbiologists, are responsible for providing reports of antimicrobial susceptibility for health care professionals (26).

Pharmacists also play a significant role in implementing the ASP $(27,28)$. Similar to other centers, in our hospital, the pharmacists were part of ASP team, and their efforts showed the significant impacts on successful implementation of team's protocol. By the aim of the involved pharmacist, the majority of whole physicians' orders were rechecked again according to ASP-prepared charts and tried to find any disagreements based on patients or laboratory's documents. According to a related study (29). Implementing ASP resulted in substantial reductions in both restricted and non-restricted antimicrobial agents, as well as changes in antimicrobial usage patterns. We found that the reduction in utilizing restricted antimicrobials was greater than non-restricted ones, possibly because physicians replaced restricted antimicrobials with nonrestricted antimicrobials based on microbiological findings of patients.

In the second year after implementing ASP, the new strategy effectively lowered restricted antimicrobials utilization and cost burden due to broad-spectrum antibiotics utilization. It should also be noted that linezolid consumption is an alarming issue, and clinicians must be consulted not to over-prescribe this antibiotic. Finally, in this study, we assessed the impact of ASP on antibiotics prescription in terms of AMR and cost burden; also, we showed the positive effect of ASP implementation and guideline-based recommendations. The main limitations of this study included low sample size, the lack of physicians' cooperation, and some missing lab data.

\subsection{Conclusions}

The current study, which is one of the first-of-its-kind reports on an ASP implementation in an Iranian pediatric 
hospital, found that ASP expenditure reduced the AMR and antimicrobial agents' prescription, especially for broadspectrum antibiotics. For maintaining the positive results, recruiting a multidisciplinary full-time ASP team including pharmacists, clinical microbiologists, and infectious disease specialists along with other educated healthcare professions is recommended.

\section{Acknowledgments}

We thank the nurses who took part in the project. Also, we thank Mrs. Soheila Torki, the hospital's supervisor of infection control, who helped us to perform the antimicrobial stewardship program (ASP).

\section{Footnotes}

Authors' Contribution: Study concept and design: A.M., Acquisition of data: I.KH., Analysis, and interpretation of data: I.KH. and N.A., Drafting of the manuscript: I.KH. and S.S, Critical revision of the manuscript for important intellectual content: A.M., Statistical analysis: I.KH. and N.A., Administrative, technical, and material support: A.M., Study supervision: A.M

Conflict of Interests: No potential conflict of interest to declare.

Data Reproducibility: The data presented in this study are openly available in one of the repositories or will be available on request from the corresponding author by this journal representative at any time during submission or after publication. Otherwise, all consequences of possible withdrawal or future retraction will be with the corresponding author.

Ethical Approval: No ethics approval was required due to the nature of the article. The protocol of study was approved by the Ethics Committee of the hospital.

Funding/Support: We did not receive any financial support.

\section{References}

1. Lesprit P, Brun-Buisson C. Hospital antibiotic stewardship. Curr Opin Infect Dis. 2008;21(4):344-9. doi: 10.1097/QCO.ob013e3283013959. [PubMed: 18594284].

2. Barlam TF, Cosgrove SE, Abbo LM, MacDougall C, Schuetz AN, Septimus EJ, et al. Executive summary: implementing an antibiotic stewardship program: Guidelines by the infectious diseases society of America and the society for healthcare epidemiology of America. Clin Infect Dis. 2016;62(10):1197-202. doi: 10.1093/cid/ciw217. [PubMed: 27118828].
3. Huttner A, Harbarth S, Carlet J, Cosgrove S, Goossens H, Holmes A, et al. Antimicrobial resistance: a global view from the 2013 World Healthcare-Associated Infections Forum. Antimicrob Resist Infect Control. 2013;2:31. doi: 10.1186/2047-2994-2-31. [PubMed: 24237856]. [PubMed Central: PMC4131211].

4. Nowak MA, Nelson RE, Breidenbach JL, Thompson PA, Carson PJ. Clinical and economic outcomes of a prospective antimicrobial stewardship program. Am J Health Syst Pharm. 2012;69(17):1500-8. doi: 10.2146/ajhp110603. [PubMed: 22899745].

5. Camins BC, King MD, Wells JB, Googe HL, Patel M, Kourbatova EV, et al. Impact of an antimicrobial utilization program on antimicrobial use at a large teaching hospital: A randomized controlled trial. Infect Control Hosp Epidemiol. 2009;30(10):931-8. doi: 10.1086/605924. [PubMed: 19712032]. [PubMed Central: PMC2779729].

6. Davey P, Marwick CA, Scott CL, Charani E, McNeil K, Brown E, et al. Interventions to improve antibiotic prescribing practices for hospital inpatients. Cochrane Database Syst Rev. 2017;2. CD003543. doi: 10.1002/14651858.CD003543.pub4. [PubMed: 28178770]. [PubMed Central: PMC6464541].

7. McCulloh RJ, Queen MA, Lee B, Yu D, Stach L, Goldman J, et al. Clinical impact of an antimicrobial stewardship program on pediatric hospitalist practice, a 5-year retrospective analysis. Hosp Pediatr. 2015;5(10):520-7. doi: 10.1542/hpeds.2014-0250. [PubMed: 26427920].

8. Newland JG, Stach LM, De Lurgio SA, Hedican E, Yu D, Herigon JC, et al. Impact prospective-audit-with-feedback antimicrobial stewardship program at a children's hospital. J Pediatric Infect Dis Soc. 2012;1(3):17986. doi: 10.1093/jpids/pis054. [PubMed: 26619405].

9. Newman RE, Hedican EB, Herigon JC, Williams DD, Williams AR, Newland JG. Impact of a guideline on management of children hospitalized with community-acquired pneumonia. Pediatrics. 2012;129(3):e597-604. doi: 10.1542/peds.2011-1533. [PubMed: 22351891].

10. Gerber JS, Newland JG, Coffin SE, Hall M, Thurm C, Prasad PA, et al. Variability in antibiotic use at children's hospitals. Pediatrics. 2010;126(6):1067-73. doi: 10.1542/peds.2010-1275. [PubMed: 21078728]. [PubMed Central: PMC4677056].

11. European Centre for Disease Prevention and Control. Summary of the latest data on antibiotic consumption in EU. Stockholm: European Centre for Disease Prevention and Control; 2017.

12. European Centre for Disease Prevention and Control. Antimicrobial resistance surveillance in Europe. Stockholm: European Centre for Disease Prevention and Control; 2015.

13. WHO Collaborating Centre for Drug Statistics Methodology. Guidelines for ATC classification and DDD assignment. Norway: WHO Collaborating Centre for Drug Statistics Methodology; 2020. Available from: https://www.whocc.no/atc_ddd_index_and_guidelines/ guidelines/.

14. Dellit TH, Owens RC, McGowan JJ, Gerding DN, Weinstein RA, Burke JP, et al. Infectious Diseases Society of America and the Society for Healthcare Epidemiology of America guidelines for developing an institutional program to enhance antimicrobial stewardship. Clin Infect Dis. 2007;44(2):159-77. doi: 10.1086/510393. [PubMed: 17173212].

15. Bratzler DW, Dellinger EP, Olsen KM, Perl TM, Auwaerter PG, Bolon $\mathrm{MK}$, et al. Clinical practice guidelines for antimicrobial prophylaxis in surgery. Am J Health Syst Pharm. 2013;70(3):195-283. doi: 10.2146/ajhp120568. [PubMed: 23327981].

16. Versporten A, Sharland M, Bielicki J, Drapier N, Vankerckhoven V, Goossens $\mathrm{H}$, et al. The antibiotic resistance and prescribing in European Children project: a neonatal and pediatric antimicrobial webbased point prevalence survey in 73 hospitals worldwide. Pediatr Infect Dis J. 2013;32(6):e242-53. doi: 10.1097/INF.0b013e318286c612. [PubMed: 23838740]. 
17. Huebner J, Rack-Hoch AL, Pecar A, Schmid I, Klein C, Borde JP. [Pilot project of a pediatric antibiotic stewardship initiative at the Hauner children's hospital]. Klin Padiatr. 2013;225(4):223-9. German. doi:10.1055/s-0033-1349063. [PubMed: 23852778].

18. Sharifi H, Pouladfar G, Shakibaie MR, Pourabbas B, Mardaneh J, Mansouri S. Prevalence of $\beta$-lactamase genes, class 1 integrons, major virulence factors and clonal relationships of multidrugresistant Pseudomonas aeruginosa isolated from hospitalized patients in southeast of Iran. Iran J Basic Med Sci. 2019;22(7):806-12. doi: 10.22038/ijbms.2019.35063.8340.

19. Izadi Pour Jahromi S, Mardaneh J, Sharifi A, Pezeshkpour V, BehzadBehbahani A, Seyyedi N, et al. Occurrence of a multidrug resistant Pseudomonas aeruginosa strains in hospitalized patients in Southwest of Iran: Characterization of Resistance Trends and Virulence Determinants. Jundishapur J Microbiol. 2018;11(4). doi: 10.5812/jjm.57341.

20. Anvarinejad M, Pouladfar G, Japoni A, Bolandparvaz S, Satiary Z, Abbasi $\mathrm{P}$, et al. Isolation and antibiotic susceptibility of the microorganisms isolated from diabetic foot infections Nemazee Hospital, Southern Iran. J Pathog. 2015;2015:328796. doi: 10.1155/2015/328796. [PubMed: 26843987]. [PubMed Central: PMC4710915].

21. Pakyz AL, Gurgle HE, Ibrahim OM, Oinonen MJ, Polk RE. Trends in antibacterial use in hospitalized pediatric patients in United States academic health centers. Infect Control Hosp Epidemiol. 2009;30(6):600-3. doi: 10.1086/597545. [PubMed: 19419328].

22. Langford BJ, So M, Raybardhan S, Leung V, Westwood D, MacFadden $\mathrm{DR}$, et al. Bacterial co-infection and secondary infection in patients with COVID-19: A living rapid review and meta-analysis. Clin Microbiol Infect. 2020;26(12):1622-9. doi: 10.1016/j.cmi.2020.07.016. [PubMed: 32711058]. [PubMed Central: PMC7832079].
23. Cook PP, Catrou PG, Christie JD, Young PD, Polk RE. Reduction in broad-spectrum antimicrobial use associated with no improvement in hospital antibiogram. J Antimicrob Chemother. 2004;53(5):853-9. doi:10.1093/jac/dkh163. [PubMed: 15044426].

24. Standiford HC, Chan S, Tripoli M, Weekes E, Forrest GN. Antimicrobial stewardship at a large tertiary care academic medical center: cost analysis before, during, and after a 7-year program. Infect Control Hosp Epidemiol. 2012;33(4):338-45. doi: 10.1086/664909. [PubMed: 22418628].

25. Beardsley JR, Williamson JC, Johnson JW, Luther VP, Wrenn RH, Ohl CC. Show me the money: long-term financial impact of an antimicrobial stewardship program. Infect Control Hosp Epidemiol. 2012;33(4):398400. doi: 10.1086/664922. [PubMed: 22418636].

26. Morency-Potvin P, Schwartz DN, Weinstein RA. Antimicrobial stewardship: How the microbiology laboratory can right the ship. Clin Microbiol Rev.2017;30(1):381-407. doi:10.1128/CMR.00066-16. [PubMed: 27974411]. [PubMed Central: PMC5217798].

27. Wickens HJ, Farrell S, Ashiru-Oredope DA, Jacklin A, Holmes A; Antimicrobial Stewardship Group of Department of Health Advisory Committee on Antimicrobial Resistance, et al. The increasing role of pharmacists in antimicrobial stewardship in English hospitals. J Antimicrob Chemother. 2013;68(11):2675-81. doi: 10.1093/jac/dkt241. [PubMed: 23825383].

28. Parente DM, Morton J. Role of the pharmacist in antimicrobial stewardship. Med Clin North Am. 2018;102(5):929-36. doi: 10.1016/j.mcna.2018.05.009. [PubMed:30126581].

29. Di Pentima MC, Chan S, Hossain J. Benefits of a pediatric antimicrobial stewardship program at a children's hospital. Pediatrics. 2011;128(6):1062-70. doi:10.1542/peds.2010-3589. [PubMed: 22106075]. 